\title{
Student Tobacco Use Behaviours: A Qualitative Study of Alternative Tobacco and Nicotine Product Use in Young Adulthood
}

\author{
(D)Aija Zobena Dr. soc. \\ University of Latvia, Latvia \\ aija.zobena@lu.lv
}

\begin{abstract}
Non-combustible alternative tobacco products such as tobacco-free nicotine pouches, heated tobacco, and electronic cigarettes (e-cigarettes) marketed as less harmful alternatives to cigarettes as smoking cessation aids are becoming increasingly popular among adolescents and young adults. This age group includes individuals still experimenting with and establishing tobacco use. The aim of the study is to investigate student tobacco use behaviours, particularly novel devices, and alternative products to understand how to decrease tobacco initiation and use among adolescents and young adults. In August 2020, two focus group discussions were organized to obtain information on young people's experience of alternative tobacco and nicotine product use. In each of them, high school students (aged over 18) and students took part. The participants of the focus group discussion were chosen by the "snowball" method. Cessation of smoking and replacing cigarettes with alternative tobacco and nicotine products reduce some of the harmful effects but are not harmless and nicotine addiction remains. By replacing cigarette smoking with the use of tobacco-free nicotine pouches, heated tobacco, or e-cigarettes, one form of nicotine use is being replaced by another. According to the study, young people have no understanding of nicotine addiction and the health risks of using alternative tobacco products. Today's adolescents and young adults often see consumption of tobacco and nicotine products as a mean to construct and project their unique identity.
\end{abstract}

Keywords: students tobacco use behaviours, alternative tobacco products, university education.

\section{Introduction}

Health Behaviour in School-aged Children (HBSC), a World Health Organization collaborative crossnational study show that despite encouraging reductions in tobacco and alcohol use since 2014, levels of current cigarette-smoking and drinking are high, particularly among 15-year-olds (Inchley et al., 2020, 29). In Latvia in 2015 the proportion of regular smokers in the 15 -year-old cohort has decreased by $12.1 \%$ and returned to the level of 1995 , when $17.0 \%$ of 15 -year-olds at that time smoked regularly (ESPAD ..., 2016, 9). However, the rapid spread of e-cigarettes and other alternative tobacco and nicotine products among adolescents and young adults is a cause for concern. Many studies show that cigarette use is still the most prevalent (Johnson et al., 2019), but alternate forms of tobacco use are particularly likely in a younger population (El Hajj et al., 2019; Ferrell et al., 2020; Dunbar et al., 2020), youth are replacing some of their cigarette smoking with other means of nicotine delivery - e-cigarettes, heated tobacco, tobacco-free nicotine pouches. Most people believe e-cigarettes to be less harmful than smoking. Previous research shows that younger people are less likely to be realistic about the risks of tobacco products and overly optimistic about their ability to quit using them (Tackett et al., 2020).

Adolescents' smoking, alcohol use and other provocative behaviours are often associated with their desire to integrate into the adult world (Ledina, Lice-Zikmane, 2020). The popularity of electronic nicotine delivery devices (such as e-cigarettes, vape pens, heated tobacco devices) among young people may be related to the perception that vapes are safer than cigarettes, interest in technological innovations containing tobacco, attractiveness for different tastes, vulnerability to peer pressure and attempts to stop smoking cigarettes. Marketing and promotion of e-cigarette devices (Peitsch et al., 2018) try to create a norm that e-cigarettes are desirable and popular, lowering the social risk of using (Marion et al., 2020). The use of these products can lead not only to continued use in adulthood, but also to cigarette smoking in people who have never smoked before.

For today's adolescents and young adults, consumption, including the use of tobacco and nicotine products, is all about showing their unique identity (Helme et al., 2020). Some authors introduce a concept of tobacco customization - the concurrent use of multiple tobacco products is practiced creating personalized tobacco 
experience matching lifestyle, culture, values, and addiction levels. "With a range of experiences made possible through an ever-increasing menu of tobacco products, flavours, and novel devices, the current youth culture of customization may also translate to tobacco customization" (Horn, Pearson, Villanti, 2016, 51). These authors explain that ". . . tobacco customization may encompass both experience and products; youth may personalize their experiences (e.g., using products to fit a variety of places and situations) including customizing their products and the ways in which they consume nicotine (e.g., configuring their own e-cigarette or hookah) and using particular products as part of social context (e.g., only using hookah with friends or cigarettes while at a bar's outdoor patio). With all tobacco product options and experiences available to youth, tobacco customization could be explained as the concurrent use of multiple tobacco products to create personalized tobacco experiences" (Horn, Pearson, Villanti, 2016, 54).

Several other studies also confirm that today's adolescents and young people often vary the use of tobacco and nicotine products according to social context and situation (Danielsson et al., 2019). Norwegian researchers point to generational differences in the use of tobacco products. In Norway and Sweden snus (Swedish moist snuff) has been used as a smoking cessation aid by many adult smokers and smokers and ex-smokers comprise most adult snus users. However, this is not true for adolescents, where larger groups of snus users have no or very limited previous tobacco experience. Recent study shows that the current pattern of tobacco use among young tobacco users in Norway seems to be very fragmented with a high occurrence of dual use (Lund, Scheffels, 2016). This indicates that the traditional divide between snus users and smokers may no longer be useful to understand tobacco use among adolescents.

In Finland, the ban on snus sales adopted in 1995 has not prevented individuals from obtaining snus through friends, personal imports, or under-the-counter sources. Results of study in Finland indicate support for the proposition that snus (Swedish moist snuff) experimentation during late adolescence is longitudinally associated with daily cigarette smoking in early adulthood, it might constitute an indicator of the propensity to proceed to regular snus use and initiation of use of other tobacco or nicotine products (Araneda et al., 2020).

Although retailers cannot sell tobacco and nicotine products to minors, products and devices are frequently accessed via social means (e.g., peers, family members) or directly purchased via online or in-person retailers. Adolescents use social sources (relatives, friends, and strangers who purchase on their behalf) more frequently than commercial sources to obtain cigarettes, vapes and other devices (Pepper et al., 2019). The market for tobacco and nicotine products in Latvia is an area where the illicit market has a large share. According to the experts' evaluation 2016 there was a significant excise duty gap on cigarettes in Latvia - 35 \% (Jurušs, Šmite-Roke, Gasūne, 2018). There are problems with the sale of such products, as consumers obtain them illegally, including online, without product quality control and age verification of the user, thus creating tax gaps. One of the factors that encourage young people to take advantage of the illegal market is the significant price differences. The author 's previous research confirms that while selling tobacco products to minors is forbidden, small shop assistants sell and "helpful" adults help adolescent people to buy these products for them (Zobena, Skrastina, 2020).

Even though the tobacco-free nicotine pad is a new product on the Latvian market, its use, especially among adolescents and young adults, is already widespread. In this study tobacco-free nicotine pouches will be called snus because that is what the discussion participants call it. The name "snus" is often used inaccurately. Snus is a traditional Swedish moist powder smokeless tobacco product. Sale of snus is illegal in all European Union countries except for Sweden and Denmark. Some EU countries like Estonia, Latvia allow the sale of tobacco-free nicotine pouches - snus-like products that contain nicotine but no tobacco. Colloquially, this product in Latvia is called snus. Liquid used in electronic cigarettes and heated tobacco in Latvia is a taxable object, but tobacco-free nicotine pouches are not. It creates differences between countries in terms and conditions of marketing and traceability of this product (O'Connell, Kephart, 2020). In Latvia the issue of tobacco-free nicotine pouches is being studied and discussed in depth at the inter-institutional level, therefore other legal restrictions may be imposed in the future.

The culture of tobacco use has changed, and the tobacco use experiences of today's youth are not the same as previous generations' experiences, making tobacco control more challenging: "Despite cigarette smoking restrictions and clean indoor air regulations, by stringing together myriad tobacco and nicotine products, it is possible for youth polytobacco users to use nicotine at school, home, work, and in social 
venues, potentially fuelling the odds of nicotine addiction or long-term tobacco use" (Horn, Pearson, Villanti, 2016, 55).

More research is needed to determine how educators' knowledge can help decrease tobacco initiation and use among school-aged children (Baera, Khoussinea, Dobbs, 2020). To increase middle school and high school educators' knowledge about tobacco products particularly novel devices and student tobacco use behaviours, it is important due to the increasing rates of use among adolescents and young adults.

The aim of the study is to investigate student tobacco use behaviours, particularly novel devices, and alternative products to understand how to decrease tobacco initiation and use among adolescents and young adults.

\section{Methodology}

The main method of data acquisition used in the study is focus group discussion. In August 2020, two focus group discussions were conducted to obtain information on experience of different - traditional and alternative - tobacco product use among young people, tobacco use patterns and attitudes toward alternative tobacco products. It was planned to invite 8 participants (smokers) and 8 participants tobacco-free pouch users - young adults (age 18-20) - to the discussion for about one and a half hours. The participants of the discussions were warned and agreed to the recording of the discussion in an audio recording. At the beginning of the discussion, it was made clear that the information obtained would be anonymized and used only in aggregate form. The discussion occurred in a free, relaxed atmosphere.

The participants of the focus group discussions were selected using the "snowball" method.

The focus group discussion included questions on how and why young people initiate smoking and use of tobacco-free nicotine pouches and other nicotine containing products. The introductory part of the discussion was devoted to smoking experience and habits - smoking experience, frequency and intensity, motivation and context (addiction, situation, pleasure), choice of tobacco products (price, taste, prestigious brands, advertising) and what other tobacco products have been tried by participants. The concluding part of the discussion focused on the awareness of young people about alternative tobacco products (e-cigarettes, heated tobacco, tobacco-free nicotine pouches).

\section{Results and Discussion}

Participants of the focus group discussions were university and high school students, majority of them were 18-21 years old. The first focus group (Table 1) was attended by young cigarette smokers.

Table 1

Participants of the 1st focus group discussion

\begin{tabular}{|l|l|l|l|}
\hline Participant & \multicolumn{1}{|c|}{ Occupation } & \multicolumn{1}{|c|}{$\begin{array}{c}\text { Smoking history } \\
\text { (years) }\end{array}$} & \multicolumn{1}{|c|}{ Smoking intensity } \\
\hline I Female 1 & $\begin{array}{l}\text { unemployed, former student, } \\
\text { plans to resume studies }\end{array}$ & 2-3 years & $\begin{array}{l}\text { in the past irregularly, in recent months } \\
\text { 3-4 cigarettes a day }\end{array}$ \\
\hline I Female 2 & university student & 4-5 years & smokes irregularly, in companies \\
\hline I Female 3 & high school student & 2-3 years & 5-10 cigarettes a day \\
\hline I Male 1 & $\begin{array}{l}\text { student, works in carpentry } \\
\text { during summer vocations }\end{array}$ & 3-4 years & about 10 cigarettes a day \\
\hline I Male 2 & student & 3-4 years & about 10 cigarettes a day \\
\hline I Male 3 & car mechanic & 4-5 years & 7-10 cigarettes a day \\
\hline I Male 4 & student & 2-3 years & smokes irregularly in company \\
\hline I Male 5 & student & 2-3 years & 5-10 cigarettes a day \\
\hline
\end{tabular}

They have recently reached adulthood and thus can buy and consume tobacco products legally. Participants of the first focus group ( 8 participants, 5 male and 3 female), discussed their smoking experiences and habits, awareness of and attitudes towards alternative tobacco products, especially tobacco-free nicotine pouches. 
The second focus group (Table 2) was attended by tobacco-free nicotine pouch (snus) users (eight participants, five male and four female), they discussed their motivation to choose this product, personal experience of using snus, and experience with using other tobacco products.

Table 2

Participants of the 2nd focus group discussion

\begin{tabular}{|l|l|l|l|}
\hline Participant & \multicolumn{1}{|c|}{ Occupation } & $\begin{array}{c}\text { Duration of snus } \\
\text { use (years) }\end{array}$ & \multicolumn{1}{|c|}{ Tobacco use experience } \\
\hline II Female 1 & art school student & 2 years & both smoke and use snus \\
\hline II Female 2 & high school student & 2 years & $\begin{array}{l}\text { previously smoked, now sometimes use IQOS or } \\
\text { very rarely smoke cigarettes }\end{array}$ \\
\hline II Female 3 & $\begin{array}{l}\text { arrived from the U.K. } \\
\text { on summer vacation }\end{array}$ & 6 months & previously used and still sometimes uses IQOS \\
\hline II Female 4 & student & 6 months & switched from smoking to snus \\
\hline II Male 1 & working in a casino & 2 years & switched from smoking to snus \\
\hline II Male 2 & project manager & 10 years & switched from smoking to snus \\
\hline II Male 3 & student & 3 years & switched from smoking to snus \\
\hline II Male 4 & project manager & 6 years & $\begin{array}{l}\text { have not smoked before, uses both tobacco } \\
\text { (snuff) and tobacco-free nicotine pouches }\end{array}$ \\
\hline
\end{tabular}

Almost all (except one) participants of both discussions have started using nicotine-containing products by smoking cigarettes. Most of the participants in the discussion were 18-21 years old. For almost all of them the first smoking experience was at the age of about 15-16, but they started regularly smoking a few years later, most often - with friends, classmates, co-workers. Participant "I female-3" told: "I am studying now in my last high school class. And I tried it [smoking] for the first time at the end of elementary school. At the time, it was so very periodic, even from the beginning in the company of friends. However, now, the effect of this quarantine could be that I started smoking a lot, much more . . I can not imagine my morning without smoking. Now, I have this routine ... you eat breakfast, and immediately demand is the desire to smoke. I also smoke about 5-10, maybe even more [cigarettes] a day."

Young people still find themselves in workplaces in situations where smoking is an essential element of informal culture. Participant "I male-3" told: “. . . I studied at a technical school as a car mechanic. I have been smoking since I was 16 years old . . I have been smoking quite actively all the time since I started. If you work more [smoke more] at work, I started smoking just when I was working ... Those who smoke, they smoke. Those who do not smoke - they work (smile)."

Almost all young people who now use tobacco-free nicotine pouches have gained their first nicotine experience from smoking. Only one focus group participant, under the influence of an older brother, has never smoked, now uses both tobacco pouches (snuff) and tobacco-free nicotine pouches.

Summarizing the opinions expressed by the participants of the discussion, it can be concluded that most often adolescents start smoking during high school, together with friends, school, or study mates. Increasingly, young people are confronted with situations where others smoke irregularly or do not smoke at all, but the participants in the discussion have not considered quitting smoking. Participant "I male-4" told: "An interesting fact ... I started my studies at Riga Stradins University . . [among] my study-mates, [there are] very few people who smoke . . from one faculty [which has] about 60 students, some 5-6 people [smoke], others do not smoke."

Young people are eager to try a variety of new tobacco and nicotine products, both roll-your-own tobacco and a variety of smokeless products, including tobacco-free nicotine pads. Nicotine intake is not always important for young people, especially if a strong addiction has not yet developed. Participant "I male-1" told: "There have also been attempts to use snus - to place inside the lip . . in other words, to get nicotine intake ... It is . . . [something like] chewing tobacco placed inside the lip and thus nicotine is obtained. As if satisfying the need [for nicotine]. And I have tried it too, and I don't see the point at all when I put it inside my lip and something stands there . . . what is the point, it seems? Cigarettes are the solution ... the process ... nicotine intake is not so important." 
Young people are very active in trying new products. Very often new products are tested on the recommendation of friends in companies when they offer them to each other. Participant "I male-2" told: "I have recently given up regular cigarettes and switched to IQOS. Because it does not smell! It does not stink like that, and the process is basically the same, and no big difference." Participant "I male-5" told: "I smoke rolling tobacco ... and I personally like the way it tastes better, and they do not stink as much as cigarettes, but it still has a little more flavour than, for example, IQOS or some electronic device . . . somehow."

In general, it can be concluded that those young people who prefer smoking daily do not show a special interest in nicotine pouches (both tobacco-containing and tobacco-free). Most young people who smoke cigarettes know about nicotine pouches, but often do not distinguish between tobacco and tobacco-free nicotine pouches. The unpleasant experience of trying tobacco pouches (snuff) discourages from trying tobacco-free nicotine pouches as well.

Users of tobacco-free nicotine pouches often find themselves trying out a variety of other nicotinecontaining products in search of the optimal way to comfortably absorb nicotine for their needs discreetly, quickly, odourless. Participant "II male-1" talked about his experience of using tobacco-free nicotine pouches: "At first I did not smoke that much . . . then I had a physical need rather than a psychological one, ... physical need for nicotine only. It is coffee, when I get up in the morning, I need nicotine to function and not be itchy, to think ... to relax, let's say after school or work, or when I need it at work. Well, yes ... that is how I started using them. I do not like to smoke, I don't like the smell, but I wanted nicotine. I tried electronic cigarettes, but they did not . . . didn't go to heart. . . . Now I stay with those pouches." Tobacco-free nicotine pouches correspond to young people's ideas about a stylish, easy-to-use product. Participant "II female-3" told: "It must be taken into account, well, that nowadays we are in a very big race ... all in this fast-paced world ... we value things for convenience, of course, if you go to smoke, it is a process... it's like a break, but let's say if you don't want a process, but you need some results, well, then ... I think people look at how comfortable they are to do something."

One of the participants of the discussion (uses nicotine pouches for 10 years) told that he has long time purposefully looking for information about nicotine pouches as a product and the opportunity to buy them. Participant "II male-2" told: "It was long time ago. . . maybe the media at that time ... [provided information about] what is used by American baseball-players what they place inside the lip ... and so we found out what it is. We found where to get it. We brought, we tried it ... Just knew that there is such American tobacco, there is also Swedish ... and that is how we brought it to Latvia". The participants of the discussion emphasized that nicotine pouches have a wide choice. Participant "II male-4" told: "Before the introduction of nicotine pads in Latvia, there was only tobacco. And there was not a large selection of these products. And now that there are nicotine pouches, I like the variety that I can choose, change those strengths, and so on. Just in the past with tobacco I had only one brand, taste, and now I can change all kinds of tastes, types and brands there." Size of the nicotine pouch is important to another participant in the discussion. Participant "II female-4" told: "For me, for example, personally, the most important thing is actually the size of the pouch ... I do not like big ones. ... Yes, I have small lips . . . So, I really like that there are small pouches, but also not too small."

Summarizing the part of the discussion about the motivation and habits of using tobacco-free nicotine pouches, it can be concluded that in the choice in favour of using this product for some participants, individual motivation to use something special, to stand out from others, is important. According to the participants of the discussion, the product must meet some special requirements - to be able to combine the use of nicotine with intense sports, to use a product that does not smell, which does not stain teeth, allows to take nicotine quickly and discreetly virtually anywhere. For the participants of the discussion, it is also important that the use of tobacco-free nicotine pouches does not pose a health risk. Quitting smoking and replacing cigarettes with smokeless products (including tobacco-free nicotine pouches) reduce some of the harmful effects, but these products also pose health risks and nicotine addiction remains. The use of tobacco-free nicotine pouches as a means of quitting smoking (and overcoming nicotine dependence by gradually reducing the nicotine dose) was not mentioned by the participants of discussion.

Three participants of discussion smoke cigarettes or use a tobacco heating device in parallel with the use of tobacco-free nicotine pouches, depending on the society in which they are staying at the time. 
In the youth environment, social smoking plays an important role, as often a severe nicotine addiction has not yet developed, so the choice of one or another product is determined by the society in which the young person is staying at that time. This confirms the usefulness of the concept of tobacco customization for understanding young adult's tobacco use behaviours. The concept of tobacco customization is essential for interpretation of expectations young people associate with consumption of different products. The use of nicotine products meets the desire of young people to customize their products and the ways in which they consume nicotine. Participant "II female-2" told: "It is more like I use pads when I'm somewhere indoors . . I I do not know . . . I'm lazy to go out somewhere or do something... You know how, differently at home when you are just lying-in bed and then you can [take snus] . . yes. And when you watch a series and you just do not want to pause. It is a much better solution in that sense, yes. Well, sometimes I also want that process - go out to smoke, sit." Another participant of the discussion "II male-4" emphasized that he is happy to buy various accessories that allow to customize the boxes for storing the pouches, to cool the pouches in the refrigerator: "I wanted to add more about those accessories, there were mentioned ... matching lighters and so on ... there is also a big market for that you can order for yourself. . custom boxes ... I remember I had my first black box ... it was made of metal, screwable, so there was another rubber here, waterproof. . . and I remember ... I always kept it in the refrigerator so that such a metal case is . . unrealistically cold. . Oh, and, yes . . . it is best to use them when they are cold . . . even before going to bed, I take them to the refrigerator, I always put them in the refrigerator so that it is cold in the morning ....".

The experience of participants of the discussion shows that a large part of nicotine products is purchased on the illegal market. Tobacco-free nicotine pouches are a new product, but legal sales of tobacco pouches are generally banned in Latvia, but many people use them and have a relatively long experience of using them. Participant "II male-4" told: "Website [where I order pouches] belongs to Latvians, the Latvian company. All buy from them . . . here in Latvia. I either go to pick them up or receive them at the parcel terminal. . they send me within a day, within two days." The main reason why tobacco-free nicotine pouches are most often purchased by discussion participants on websites or with friends is price. When ordering on the website in bulk, the price difference is significant, so it is often ordered jointly. Participant "II male-3" told: "I also buy them from friends . . . in support of the shadow economy (laughs). It is very easy to find people who sell snus banks. . . . This is their full-time job. Of course, [I can buy them] at more adequate prices than in the store."

In general, participants of the discussion support legal sale of tobacco-free nicotine pouches, Participant "II female-4" told: "It seems to me that it is better that it is allowed, it is regulated than it is not allowed. People would also use it, but it would not be regulated. It will not be possible to control. It is better to control it than it just is [bought illegally]."

\section{Conclusions}

Most young smokers are convinced that they can control the intensity of smoking, do not think much about the negative consequences of smoking, they have not seriously considered smoking cessation. Interest in new products, which are positioned in the market as less harmful and as a means of quitting smoking, is more often related to the desire to try new things to experiment with new feelings.

In general, other smokeless tobacco products are more attractive to smokers than tobacco-free nicotine pouches. At present, tobacco heating devices are topical, while the interest in e-cigarettes for young people is declining.

By replacing cigarette smoking with the use of tobacco-free nicotine pouches, one form of nicotine use is replaced by another. Young people have no understanding of nicotine addiction and the health risks of using nicotine pouches.

In general, it can be concluded that those young people who prefer smoking daily do not show special interest in nicotine pouches (both tobacco-containing and tobacco-free). Most young people who smoke cigarettes are aware of tobacco-free nicotine pouches, but often do not distinguish between tobacco and tobacco-free nicotine pouches. The disgusting experience of trying tobacco pouches (snuff) discourages them from trying tobacco-free nicotine pads as well. 
The first, even negative, experience of using tobacco-free nicotine pouches for young people who use snus did not deter them from trying again. This has been facilitated by the transfer of knowledge and experience within the subculture of tobacco-free nicotine pouch users.

Users of tobacco-free nicotine pouches are aware of the difference between tobacco (snuff) and tobaccofree nicotine pouches. Tobacco-free nicotine pouches are perceived as a higher quality, cleaner and more pleasant product to use.

Some participants practiced use of multiple tobacco products creating personalized tobacco experiences matching lifestyle, culture, values and addiction levels.

Discussion participants usually order tobacco-free nicotine pouches on websites (buy online) or from friends ("buy from hand"), where they can be bought much cheaper than at points of sale in supermarkets, gas stations. Price differences between the legal and illegal markets are significant, so most of the participants in the discussion are already buying snus and snuff from illegal distributors.

Participants believe that if the sale of tobacco-free nicotine pouches is legal and controllable, consumers are confident in the quality of the products. However, if tobacco-free nicotine pouches are banned from being sold legally, users will be able to buy what they want by ordering online or on the black market.

\section{Bibliography}

1. Araneda D., Korhonen T., Laatikainen T., Haukkala A., Rose R.J., Kaprio J. (2020). Association of snus experimentation in late adolescence with daily cigarette smoking in early adulthood: A longitudinal study among Finnish men. Scandinavian Journal of Public Health, 48(6), 638-645. doi: 10.1177/1403494819828868

2. Baera C.M., Khoussinea M.A., Dobbs, P.D. (2020). Tobacco prevention education for middle school and high school educators. Health Education Journal, 1-12. doi: 10.1177/0017896920950344

3. Danielsson M., Lammi A., Siitonen S., Ollgren J., Pylkkänen L., Vasankari T. (2019). Alarming development of dual snus and cigarette usage among young Finnish males. BMC Public Health, 19, 1249. doi: 10.1186/s12889-019-7519-1

4. Dunbar M.S., Davis J.P., Tucke J.S., Seelam R., Shih R.A., D’Amico E.J. (2020). Developmental Trajectories of Tobacco/Nicotine and Cannabis Use and Patterns of Product Co-use in Young Adulthood. Tobacco Use Insights, 13, 1-10. doi: 10.1177/1179173X20949271

5. El Hajj D., Cook P.F., James K.A., Battaglia C., Prochazca A.V. (2019). Newer Forms of Tobacco Products: Characteristics of Poly Users Among Adults Living in Colorado-A Secondary Data Analysis of the Attitudes and Behaviors Survey on Health 2015. Tobacco Use Insights, 12, 1-6. doi: 10.1177/1179173X19874811

6. ESPAD 2015 - Atkarību izraisošo vielu lietošanas paradumi un tendences skolēnu vidū [European School Survey Project on Alcohol and Other Drugs - Addictive substance use habits and trends among schoolboys]. (2016). Rīga: SPKC, 9. Retrieved from https://www.spkc.gov.lv/lv/petijumi/atkaribu-izraisoso-vielu-lietosanas-paradumi-un-tendencesskolenu-vidu.-espad-2015.pdf (in Latvian)

7. Ferrell A., Hadddad L., Elder J.H., Garvan C., Cook C.L., Salloum R. (2020). Perceptions and Use of Electronic Nicotine Delivery Systems Among Floridian Middle and High School Students: Secondary Analysis of Cross-sectional Survey Results. Tobacco Use Insights, 13, 1-7. doi: 10.1177/1179173X20953402

8. Helme D.W., Morris E., de la Serna A., Zelaya C., Oser, C., Knudsen, H.K. (2020). "Country Boys Spit and Dip": Masculinity and Rural Adolescent Smokeless Tobacco Use. Journal of Men's Studies, 1-22. doi: 10.1177/1060826520946458

9. Horn K., Pearson J.L., Villanti A.C. (2016). Polytobacco Use and the "Customization Generation" - New Perspectives for Tobacco Control. Journal of Drug Education, 46(3-4), 51-63. doi: 10.1177/0047237917722771

10. Inchley J., Currie D., Budisavljevic S., Torsheim T., Jåstad A., Cosma A., Kelly C., Arnarsson Á.M. (Eds.). (2020). Spotlight on adolescent health and well-being. Findings from the 2017/2018 Health Behaviour in School-aged Children (HBSC) survey in Europe and Canada. International report. 
Volume 1. Key findings. Copenhagen: WHO Regional Office for Europe. Retrieved from https://apps.who.int/iris/bitstream/handle/10665/332091/9789289055000-eng.pdf

11. Johnson A.L., Villanti A.C., Williams V., Rath J.M., Vallone D.M., Abrams D.B., Hedeker D., Mermelstein R.J. (2019). Smoking Trajectory Classes and Impact of Social Smoking Identity in Two Cohorts of U.S. Young Adults. Emerging Adulthood, 7(4) 258-269. doi:10.1177/2167696818763949

12. Jurušs, M., Šmite-Roķe, B., Gasūne, L. (2018). Excise Duty Gap on Cigarettes. Inzinerine Ekonomika-Engineering Economics, 2018, 29(4), 419-423. doi: 10.5755/j01.ee.29.4.20335

13. Ledina I., Lice-Zikmane I. (2020). The Pedagogical Aspects of Reducing Adolescent Violence in the School Environment. In V. Dislere (Ed.), The Proceedings of the International Scientific Conference Rural Environment. Education. Personality (REEP), 13. Jelgava: Latvia University of Life Sciences and Technologies, 303-309. doi: 10.22616/REEP.2020.036

14. Lund I., Scheffels J. (2016). Adolescent tobacco use practices and user profiles in a mature Swedish moist snuff (snus) market: Results from a school-based cross-sectional study. Scandinavian Journal of Public Health, 44(7), 646-653. doi: 10.1177/1403494816656093

15. Marion H., Garner W., Estrada A., Moorer C., Acosta-Velazquez I. (2020). Online Pro-Tobacco Marketing Exposure Is Associated with Dual Tobacco Product Use Among Underage US Students. American Journal of Health Promotion, 4(6) 648-651. doi:10.1177/0890117120905231

16. O'Connell M., Kephart L. (2020). Local and State Policy Action Taken in the United States to Address the Emergence of E-Cigarettes and Vaping: A Scoping Review of Literature. Health Promotion Practice. Onlinefirst. doi: 10.1177/1524839920963691

17. Peitsch M.C., Polosa R., Proctor C., Hassler T., Gaca M., Hill E., Hoeng J., Hayes A.W. (2018). Next-generation tobacco and nicotine products: Substantiating harm reduction and supporting tobacco regulatory science. Toxicology Research and Application, 2, 1-12. doi: $10.1177 / 2397847318773701$

18. Pepper J.K., Coats E.M., Nonnemaker J.M., Loomis B.R. (2019). How do adolescents get their e-cigarettes and other electronic vaping devices? American Journal of Health Promotion, 33(3), 420-429. doi: 10.1177/0890117118790366

19. Tackett A.P., Keller-Hamilton B., Hébert E.T., Smith C.E., Wallace S.W., Stevens E.M., Johnson A.L., Wagener T.L. (2020). Adolescent Susceptibility to E-Cigarettes: An Update From the 2018 National Youth Tobacco Survey. American Journal of Health Promotion doi: $10.1177 / 0890117120971121$

20. Zobena A., Skrastina A. (2020). Starting and Maintaining Smoking: A Qualitative Study of Tobacco Use in High School Students. In V. Dislere (Ed.), The Proceedings of the International Scientific Conference Rural Environment. Education. Personality (REEP), 13. Jelgava: Latvia University of Life Sciences and Technologies, 348-355. doi: 10.22616/REEP.2020.042 